\title{
Desafios das Interfaces Gestuais para a Aprendizagem de Pessoas com Dispraxia
}

\author{
Leonardo Ramon Nunes de Sousa ${ }^{1,2}$, Ismar Frango Silveira ${ }^{2}$ \\ ${ }^{1}$ Universidade Federal do Piaui (UFPI) \\ Centro de Educação Aberta e a Distância (CEAD) \\ Teresina - PI - Brasil \\ ${ }^{2}$ Universidade Presbiteriana Mackenzie (UPM) \\ Programa de Pós-Graduação em Engenharia Elétrica e Computação (PPGEEC) \\ São Paulo - SP - Brasil \\ \{leonardoramon, ismarfrango\}@gmail.com
}

\begin{abstract}
Gestural interfaces-based computational tools can help people with Dyspraxia as a resource for their learning improvement. This paper discusses the research challenges related to the development of touchless applications for Special Education. The aspects of interface design, accessibility, guidelines, financial impact and the cognitive aspects are discussed, having as a target the use of assistive technologies to provide the same learning conditions for all, being in collaborative or individualized learnings contexts.
\end{abstract}

Resumo. Ferramentas computacionais que se utilizam de interfaces gestuais podem ajudar as pessoas com Dispraxia como um recurso para melhoria de sua aprendizagem. Este trabalho tem como objetivo discutir os desafios de pesquisa relacionados ao desenvolvimento de aplicativos touchless para a Educação Especial, juntamente com questionamentos sobre o desenho da interface, acessibilidade, criação de diretrizes, impacto financeiro e aspectos cognitivos a serem mobilizados, almejando-se um aluno com igualdade de condições de aprendizagem quer em contextos de aprendizagem colaborativa ou individualizada através de Tecnologias Assistivas.

\section{Introdução}

Há uma diversidade de recursos tecnológicos que podem ajudar pessoas com necessidades educacionais especiais, sendo que conhecê-los apropriadamente é um desafio para os profissionais desta área. Se for particularizado para indivíduos comprometidos com os movimentos, percepções, pensamentos e a linguagem — os dispráxicos, as tecnologias que se utilizam de interfaces gestuais podem agilizar como meio de alfabetização, aprendizagem e comunicação.

Para tanto, necessita-se discutir os desafios necessários aos aplicativos voltados para pessoas com Dispraxia ao se utilizarem dessas interfaces, com investigações aprimoradas para os próximos anos, já que atuariam como perspectivas de qualidade para a Educação Especial e que a comunidade brasileira de Informática na Educação contribuiria para discussão e reflexão.

Outros aspectos também precisam ser detalhados e discutidos, tais como custo financeiro ao se desenvolverem aplicações e dispositivos que suportem tal tecnologia, sua 
acessibilidade, impactos, nível de complexidade e aspectos cognitivos requeridos para pessoas com Dispraxia ao se utilizarem de tais recursos, além das diretrizes que serão criadas ou melhoradas.

O artigo encontra-se assim organizado: a Seção 2 aborda as tecnologias assistivas e as pessoas com deficiência, ressaltando as características das interfaces gestuais e da Dispraxia. Na Seção 3, apresenta-se o panorama geral da pesquisa de forma a situar o estado da arte, os desafios existentes e a área de trabalho a ser destacada como futuro.Por fim, na 4 são colocadas as Considerações Finais e potencialidades para os próximos estudos.

\section{Tecnologias Assistivas e Pessoas com Deficiência}

Terminologia originária do inglês Assistive Technology, a Tecnologia Assistiva (TA) é toda estratégia, recurso ou ferramenta, promovendo independência e autonomia para a Pessoa com Deficiência (PcD), além de qualidade de vida, inclusão educacional, digital e social [Cardoso et al. 2014]. Proporciona também ampliação de sua comunicação, igualdade de condições e de mobilidade, desenvolvimento de habilidades e integração com a família, amigos e sociedade.

De acordo com [Kleina 2012], TA podem ser bengalas, lupas, óculos, teclados em Braille, sintetizadores de voz, sistemas para reconhecimento de voz ou sistemas computadorizados em nível de hardware ou software, permitindo interações para combater os impedimentos linguísticos, motores ou sensoriais das $\mathrm{PcD}$, podendo ser categorizadas como: (i) adaptações em veículos; (ii) adequação postural; (iii) auxílios de mobilidade, para a vida diária e para a vida prática, para cegos ou para pessoas com visão subnormal e para surdos ou défict auditivo; (iv) comunicação aumentativa e alternativa (CAA); (v) projetos arquitetônicos para acessibilidade; (vi) próteses e órteses; (vii) recursos de acessibilidade ao computador e (viii) sistemas de controle ao ambiente.

Em relação a Pessoa com Deficiência, o termo "deficiência"significa uma restrição física, mental ou sensorial, de natureza permanente ou transitória, que limita a capacidade de exercer uma ou mais atividades essenciais da vida diária, causada ou agravada pelo meio econômico e social [CONVENÇÃO 2006], sendo categorizada como de altas habilidades, deficiência auditiva, distúrbios de conduta e deficiência intelectual.

No Brasil particularmente, a população com deficiência chega a 23,9\% do total, representando mais de 45 milhões de pessoas [IBGE 2010], sendo que a Constituição Federal garante o direito a educação e dever do Estado oferecê-la para o pleno desenvolvimento da pessoa, seu preparo para a cidadania e sua qualificação para o trabalho, sem distinção de raça, cor ou idade [BRASIL 1988], cabendo ao Estado Brasileiro garantir as $\mathrm{PcD}$ o acesso à educação, tanto a escolar regular como também a utilização de novas tecnologias, para que aconteça o processo de inclusão digital, acompanhando os avanços pela promoção de ações e políticas para conhecimento dos recursos digitais existentes através de TA, já que estas se propõem a gerar igualdade de condições aos sujeitos.

\subsection{Interface Gestual}

A comunicação sem contato é uma forma de relação com sistemas computacionais sem interação física ou mecânica de dispositivos periféricos, como o mouse, teclado, voz ou toque pela superfície da tela (touchscreen). Esse processo interativo mais natural se dá 
através de interfaces - denominadas gestuais ou touchless, que reconhecem gestos, expressões ou partes do corpo humano, como mãos, olhos, voz, pulsos elétricos emitidos pelo cérebro, músculos ou nervos, podendo ocorrer também a combinação de várias partes [Salim 2014]

Ao se utilizar as mãos como forma de reconhecimento dos gestos por essas interfaces, por exemplo, necessita-se do mapeamento das orientações e posições para que um sistema digital identifique que tarefas devem ser executadas, através de softwares e de dispositivos de hardware como antenas, bastões, braceletes, câmeras, luvas, microfones e sensores de postura, presença ou profundidade [Norman and Nielsen 2010].

As interfaces touchless são exemplos de TA por ajudarem na comunicação e interação das PcD. Especificamente, elas não são recomendadas para deficientes visuais em virtude das limitações que estes sujeitos têm e pela necessidade da visão para executálas, mas podem gerar vantagens significativas de qualidade de vida para os demais.

O reconhecimento e rastreamento de objetos nasceu na área de Visão Computacional com o desenvolvimento de sistemas baseados na detecção dos dedos, como no trabalho pioneiro de [Queck et al. 1995], e na área de detecção de tom de pele [Kulessa and Hoch 1998]. Atualmente estão presentes desde dispositivos portáteis até as tecnologias direcionadas a jogos eletrônicos, facilitando a execucção das atividades cotidianas.

\subsection{Dispraxia}

A Dispraxia, também conhecida como Transtorno do Desenvolvimento da Coordenação (TDC), é um distúrbio ligado à coordenação motora que compromete no rendimento escolar, a educação física e nas atividades cotidianas como se vestir, higiene pessoal, alimentação, relações sociais e saúde, sendo que não há lesão no cérebro clinicamente evidente. Ela é caracterizada principalmente por dificuldades espacial, motora, postural e verbal, comprometendo os movimentos, percepções, pensamentos e a linguagem [Portwood 2013].

Na espacial, há confusão nos conceitos de alto, baixo, longe ou perto, assim como na parte escrita com as formas e tamanhos de figuras. Como disfunção motora neurológica, o TDC impede o cérebro de desempenhar todas suas funções, comprometendo o equilíbrio, gerando imprecisão e lentidão. As áreas que sofrem mais alterações são as do esquema corporal e a orientação têmporo-espacial. A postura se reflete em movimentos sem ritmo e com pouco controle. Em alguns casos, a linguagem não é afetada, mas existe déficit fonológico e fonético na fala. As principais características deste transtorno podem ser verificadas na Figura 1.

A criança com Dispraxia apresenta fracasso escolar, pois a escrita manual é a área mais comprometida em virtude da dificuldade em controlar e segurar o lápis, pela pouca sensação tátil e pela noção espacial comprometida, sendo esta caracterizada pela ausência de espaçamento entre as letras e pelo impedimento em posicionar o lápis em um ponto específico do papel, juntamente com a falta de percepção tridimensional como copiar ou desenhar figuras geométricas e uma desorganização na apresentação de trabalhos no papel [Miyahara and Möbs 1995]. Usando tecnologias digitais, no entanto, os problemas escolares podem ser superados, já que a parte cognitiva não é afetada e as crianças com dispraxia podem utilizá-las com destreza e rapidez. 


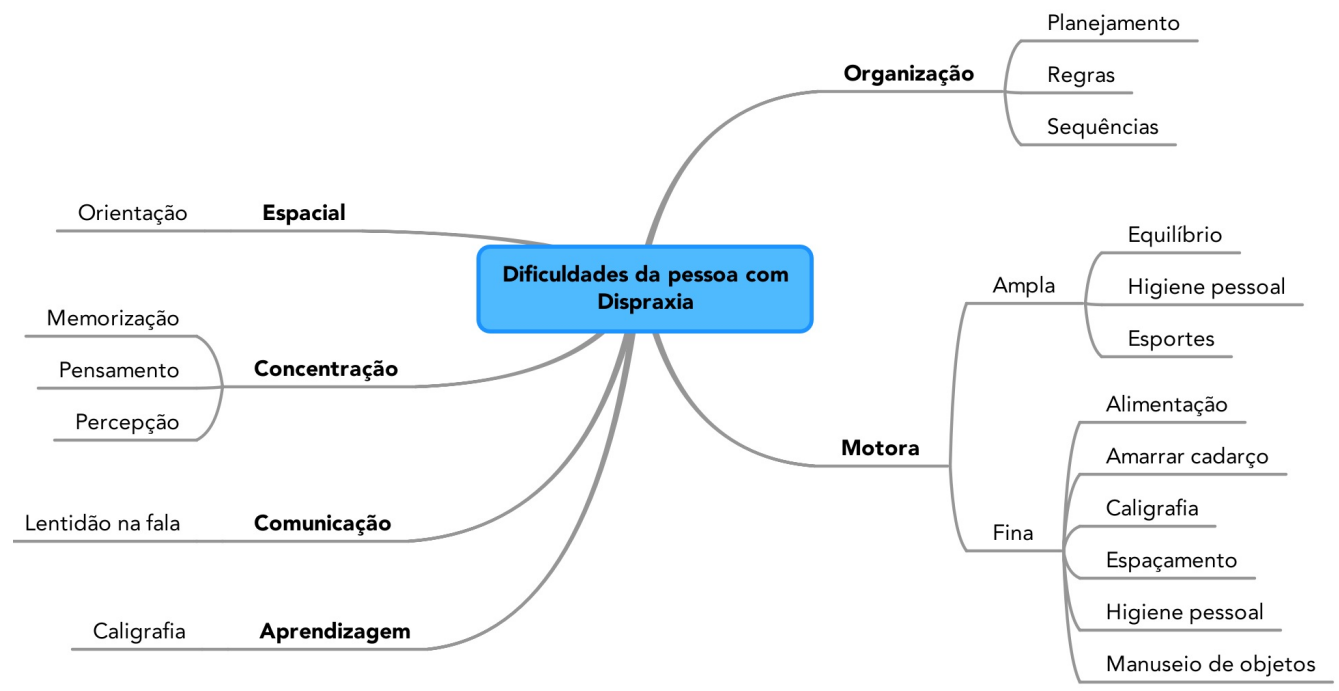

Figura 1. Dispraxia: Dificuldades e Sintomas.

Existem formas tradicionais de identificar e avaliar o TDC por profissionais especializados (educadores de psicomotricidade, fisioterapeutas, fonoaudiólogos, médicos e terapeutas ocupacionais), utilizando-se de conhecimentos tais como o Movement $A B C$ Battery for Children [Henderson et al. 1992] e o Developmental Co-ordination Disorder Questionnaire [Crawford et al. 2001], em que este último compara três testes diferentes, mostrando suas complexidades e descobrindo que as avaliações não identificavam adequadamente as crianças como tendo ou não Dispraxia. Outros recursos também podem ser utilizados em intervenções, terapias e treinamentos através de vídeos com brincadeiras, como os Handwriting Without Tears [Olsen et al. 2001] e Tree Fu Tom [Foulder-Hughes 2015].

Aprendizagem em crianças com Dispraxia deve ser enfocada durante a alfabetização, na sua parte de caligrafia e letramento, com técnicas e ferramentas que melhorem aspectos físicos e psicológicos da criança nesta etapa [Othman and Keay-Bright 2010], proporcionando mais autonomia e segurança, como exercícios pontilhados, utilização de massa de modelar atóxica, tabuleiros, quadros de areia, giz ou pincel, cadeiras e mesas adaptadas, diferentes tipos de lápis, canetas - as que acendem uma luz quando pressionadas, borrachas, réguas, papel com pauta e que esteja sempre alinhado com o braço da criança.

Pode-se permitir também que a escrita da criança seja em letra de forma, exigir quantidade determinada de trabalhos ou exercícios, fazendo-a usufruir de outros momentos de interação com os colegas, como os intervalos, além de dar tempo extra, não marcar todos os erros em seu material ao corrigí-los, incentivar respostas orais ou a utilização de tecnologias digitais que usufruem de movimentos cinéstesicos - aqueles realizados no ar, como as tecnologias que se utilizam de interfaces gestuais, ajudando no processo de aprendizagem de crianças com Dispraxia, já que estas têm problemas em finalizar e inverter letras (lateralidade e orientação). 


\section{Panorama da Pesquisa}

Como exemplos de aplicativos existentes no mercado que trabalham com interfaces touchless, pode-se conferir a Tabela 1 - criada a partir de uma Pesquisa Exploratória e Documental [Gil 2002], que ressalta os desafios, potencialidades e limitações de cada tecnologia. Estas aplicações podem atuar na reabilitação e em intervenções de distúrbios, necessitando-se que sejam aperfeiçoadas e adaptadas para utilização na alfabetização ou aprendizagem de pessoas com Dispraxia.

Tabela 1. Caracterizações e Desafios em Tecnologias Gestuais.

\begin{tabular}{|c|c|c|c|}
\hline $\begin{array}{l}\text { Tecnologia } \\
\text { Gestual }\end{array}$ & Potenciais & Limitações & Desafios \\
\hline $\begin{array}{l}\text { ASUS Xtion } \\
\text { Motion Sensor }\end{array}$ & $\begin{array}{l}\text { Facilidade de aprendiza- } \\
\text { gem colaborativa através } \\
\text { do acesso às redes soci- } \\
\text { ais; Compatibilidade com } \\
\text { jogos existentes. }\end{array}$ & $\begin{array}{l}\text { Desativação do mouse; } \\
\text { Resposta aos comandos } \\
\text { com atraso significativo; } \\
\text { Navegador próprio pro- } \\
\text { blemático; Aumento das } \\
\text { funcionalidades para além } \\
\text { do entretenimento. }\end{array}$ & $\begin{array}{l}\text { Integração do mouse com } \\
\text { o sensor de captura ges- } \\
\text { tual; Diminuição do tempo } \\
\text { da resposta de atraso; Me- } \\
\text { lhora do navegador nas re- } \\
\text { des sociais. }\end{array}$ \\
\hline $\begin{array}{l}\text { GestureTek - } \\
\text { Projeções Inte- } \\
\text { rativas (Parede, } \\
\text { Piso) }\end{array}$ & $\begin{array}{l}\text { Estimulação e interativi- } \\
\text { dade (ação, diversão e mo- } \\
\text { vimento); Utilização na } \\
\text { educação e em eventos } \\
\text { (comerciais ou sociais). }\end{array}$ & $\begin{array}{l}\text { Sensores de reconheci- } \\
\text { mento imprecisos. }\end{array}$ & $\begin{array}{l}\text { Melhoria na precisão dos } \\
\text { sensores. }\end{array}$ \\
\hline Leap Motion & $\begin{array}{l}\text { Ajuda no processo de } \\
\text { alfabetização, apren- } \\
\text { dizagem através dos } \\
\text { movimentos cinestésicos e } \\
\text { memorização na formação } \\
\text { das letras e números. }\end{array}$ & $\begin{array}{l}\text { Necessidade de muita cali- } \\
\text { bragem e habilidades com } \\
\text { gestos. }\end{array}$ & $\begin{array}{l}\text { Melhorar a calibragem e o } \\
\text { reconhecimento dos movi- } \\
\text { mentos das mãos. }\end{array}$ \\
\hline $\begin{array}{l}\text { Microsoft Ki- } \\
\text { nect (Xbox } \\
\text { 360) - Win And } \\
\text { I }\end{array}$ & $\begin{array}{l}\text { Reconhecimento do } \\
\text { usuário (facial, perfil e } \\
\text { voz); Bom para exercícios, } \\
\text { brincadeiras e terapias. }\end{array}$ & $\begin{array}{l}\text { Não reconhece timbres de } \\
\text { voz, movimentos lentos, } \\
\text { instáveis e imprecisos. }\end{array}$ & $\begin{array}{l}\text { Melhorar a precisão dos } \\
\text { movimentos e foco maior } \\
\text { nos dedos. }\end{array}$ \\
\hline $\begin{array}{l}\text { MYO Armband } \\
\text { (Thalmic Labs) }\end{array}$ & $\begin{array}{l}\text { Detecta a contração dos } \\
\text { músculos dos braços; } \\
\text { Não exige área pré- } \\
\text { determinada para detecção } \\
\text { dos movimentos. }\end{array}$ & $\begin{array}{l}\text { Alcance de } 100 \mathrm{~m} ; \text { Pulseira } \\
\text { com tamanho único. }\end{array}$ & $\begin{array}{l}\text { Aumento do raio de al- } \\
\text { cance do dispositivo e } \\
\text { disponibilização de pul- } \\
\text { seiras com tamanhos } \\
\text { adaptáveis. }\end{array}$ \\
\hline $\begin{array}{l}\text { Nintendo Wii } \\
\text { (U) }\end{array}$ & $\begin{array}{l}\text { Boa sensibilidade aos mo- } \\
\text { vimentos; Jogos colabora- } \\
\text { tivos. }\end{array}$ & $\begin{array}{l}\text { Conexão à Internet; Auto- } \\
\text { nomia do console (game- } \\
\text { pad); Gráficos e imagens } \\
\text { desejáveis. }\end{array}$ & $\begin{array}{l}\text { Melhoria no modo online, } \\
\text { nos controles, gráficos } \\
\text { e imagens; Aumento do } \\
\text { tempo de vida da bateria. }\end{array}$ \\
\hline $\begin{array}{l}\text { PlayStation } \\
\text { Move e Eye } \\
\text { (Sony) }\end{array}$ & $\begin{array}{l}\text { Imersão; Jogos em grupo; } \\
\text { Exercícios físicos. }\end{array}$ & $\begin{array}{l}\text { Lento quando logado na } \\
\text { Internet; Similar ao Wii; } \\
\text { Muito sensível aos movi- } \\
\text { mentos; Calibração em } 3 \\
\text { passos. }\end{array}$ & $\begin{array}{l}\text { Melhoria no processamento } \\
\text { e reconhecimento dos } \\
\text { gestos; Retirada do bastão } \\
\text { (controle); Calibração; } \\
\text { Preço. }\end{array}$ \\
\hline $\begin{array}{l}\text { Wisee: } \quad \text { Wi-Fi } \\
\text { signals }\end{array}$ & $\begin{array}{l}\text { Agilidade nas tarefas co- } \\
\text { tidianas; Interações ba- } \\
\text { seadas em gestos para } \\
\text { aplicações do contexto re- } \\
\text { sidencial. }\end{array}$ & $\begin{array}{lr}\text { Comandos } & \text { embuti- } \\
\text { dos nos equipamentos; } \\
\text { Manutenção; } \\
\text { constantes. }\end{array}$ & $\begin{array}{l}\text { Sinal sempre bom; Melhora } \\
\text { na calibração. }\end{array}$ \\
\hline
\end{tabular}




\subsection{Estado da Arte}

O escopo de pesquisa em TA contém abordagens bem abrangentes, seja pelo conceito destes recursos ou por seus enfoques nas diferentes classificações de deficiência. Em [McKnight and Davies 2012] há uma revisão sobre TA para aprendizagem e relações com algumas deficiências como Transtorno do Espectro do Autismo (TEA), Deficiência Auditiva, Motora e Visual, Dislexia, Discalculia, Dispraxia e Transtorno do Défict de Atenção e Hiperatividade (TDAH), levando-se em consideração três aspectos: o aprendiz, a tecnologia e o contexto educacional.

Este trabalho, portanto, fornece uma visão do que é estudado, as revistas com materiais relevantes e nichos de pesquisa para exploração. Especificamente em relação a Dispraxia, os estudos de [Othman and Keay-Bright 2010] e [Othman and Keay-Bright 2011] usam técnicas de animação para o desenvolvimento de habilidades de caligrafia.

Existem também estudos que mencionam frameworks para o desenvolvimento e utilização de TA voltados para acessibilidade das PcD, tais como [Clarke et al. 2011], [Bohman and Anderson 2005] e [CAST 2012]. Outros como [Placitelli and Gallo 2012] enfocam interfaces gestuais, podendo-se citar os estudos de [Erazo and Pico 2014] como modelo de revisão bibliográfica recente sobre touchless, [Cohn et al. 2011] com sensores de gestos em antenas e [Mitra and Acharya 2007] como um trabalho clássico. Utilizando Kinect, têm-se os trabalhos de [Chen et al. 2014] e [Erazo et al. 2014]. Já usando o LeapMotion, há o [Shen et al. 2014].

Alguns trabalhos enfocam a criação de guidelines para a criação de aplicativos de modo geral, aos direcionados a determinados transtornos ou para aqueles publicados em lugares específicos. [Abbott 2007] lança diretrizes para pesquisadores no campo de TA publicadas no King's College London e novamente [CAST 2012] enumera princípios divididos em categorias para suporte a aprendizagem. Já [Davis et al. 2010] criaram diretrizes para conduzir o desenvolvimento de softwares direcionados a pessoas com TEA. O trabalho de [BDA 2012] trata de diretrizes para pessoas com Dislexia. Em [McKnight 2010] há guidelines para pessoas com TDAH. Em [Feng et al. 2010] mencionam guias de recomendações para desenvolvimento web atendendo as especificidades de crianças com Síndrome de Down. E [Falcão and Price 2010] ressaltam diretrizes de desenvolvimento voltadas para pesquisas em crianças com dificuldade de aprendizagem.

Esses frameworks e guidelines têm possibilidades de auxiliar na criação de interfaces gestuais voltadas para $\mathrm{PcD}$, programando, transformando ou analisando dados manipulados, classificando ou carregando gestos, gravando e possibilitando treinos, além de expandir a capacidade de reconhecimento gestual. E como restrições, eles podem atuar somente como armazenadores de códigos e métodos teóricos, havendo necessidade de incentivo para utilização, desenvolvimento e referenciamento constantes.

\subsection{Desafios Existentes}

Outros aspectos em relação à tecnologia touchless na EE precisam ser investigados com maior profundidade, como, por exemplo, o custo financeiro ao se desenvolverem aplicações e dispositivos que suportem tal tecnologia. Seu valor será acessível? Quais os impactos? Além de outras perguntas, tais como: Qual o nível de complexidade necessário para pessoas com Dispraxia utilizarem tais recursos? Que aspectos cognitivos serão mobilizados? O que se pretende discutir? Quais guidelines serão criadas e melhoradas para 
se desenvolverem aplicativos touchless para pessoas com Dispraxia? Estes dispositivos funcionam efetivamente para pessoas com TDC?

Em relação ao desenho da interface em IHC, não se pode ignorar os princípios da interação [Nielsen et al. 2004] [Norman and Nielsen 2010], assim como algumas perguntas sobre a determinação da intencionalidade gestual, por exemplo: que gestos devem ser utilizados? Quais gestos estão associados a quais ações? Ou quais comandos devem ser executados com que gestos? Qual o espaço de interação entre os usuários ou entre ele e o dispositivo? Qual o tempo de resposta (feedback) para uma ação ser executada pelo sistema digital e necessitar de repetição em caso de insucesso? Qual o tempo de calibração? E de permanência no ar? E o tempo de relaxamento das mãos do usuário? Há necessidade de movimentos com muita precisão?

Outras questões deveriam investigar o motivo pelo qual todos os aplicativos mencionados na Tabela 1 não são difundidos em $\mathrm{PcD}$, percebendo-se que a maioria deles foram projetados para pessoas sem problema motor, incorporando-se como um desafio na área de interfaces gestuais como ajuda para a aprendizagem na Educação Especial.

\section{3. Área de Trabalho}

De forma a utilizar TA para pessoas com Dispraxia, um nicho a ser explorado é o desenvolvimento de aplicações que utilizam interfaces gestuais, sem a necessidade do mouse ou do toque em alguma superfície em um dispositivo eletrônico para reconhecer um determinado comando. E ao se desenvolver aplicativos touchless, ou seja, que utilizam interfaces baseadas em gestos realizados com a mão e sem contato, recomenda-se a participação de diferentes profissionais e usuários (design participativo), sendo necessário um conjunto de diretrizes ou guidelines sobre como desenvolver estas aplicações, avaliá-las pelos usuários ou através de modelos e para que haja o envolvimento de pessoas com este distúrbio durante todo o procedimento, que elas tenham a melhor utilização possível e obedeçam às necessidades básicas destes sujeitos.

Analisando-se nas perspectivas educacional, de acessibilidade e usabilidade, estas guidelines são necessárias de forma a obedecerem aos princípios do Design Universal ou desenho da interface, dando as mesmas oportunidades de aprendizagem a todos, sendo diversificada e inclusiva, contribuindo para múltiplos significados de representação, expressão, ação e engajamento, seguindo também as normas da ONU em sua Convenção dos Direitos das Pessoas com Deficiência [UNESCO 2006].

Ainda para a área de Interface Humano-Computador (IHC), têm-se ganhos e inovações na parte de manipulação dos recursos computacionais através do reconhecimento de gestos sem toque e com desafios sob o ponto de vista da interface com as etapas de desenvolvimento de sistemas para interpretação de gestos, imagens e sons, podendo promover uma maior interação social, digital e de comunicação, aumentando a participação de pessoas com Dispraxia em ações colaborativas, organizacionais e de planejamento, além de ajudar nos processos de tratamento com intervenções em crianças com risco de marginalização.

É interessante ressaltar a ajuda que essas interfaces gestuais fornecem na Educação Especial como ambiente educacional ao promoverem a prática de movimentos cinestésicos pelas pessoas com TDC, pois são realizados no ar, repetindo modelos, reforçando a aprendizagem e a fixação visual de como uma letra é formada, contribuindo, 
assim, no processo de alfabetização, caligrafia e letramento. Além disso, ela tende a ser compensatória, promovendo motivações, aumentando habilidades de comunicação e diversificando a criação de ambientes de aprendizagem interativos e com potencial de ensino individualizado diferenciado.

Neste sentido, este trabalho chama a atenção da área de Informática na Educação para os desafios existentes em aplicativos voltados para a Educação Especial ao se utilizarem de interfaces gestuais como meio de alfabetização, aprendizagem e comunicação.

\section{Considerações Finais}

De acordo com o apresentado ao longo do texto, é fundamental que as interfaces gestuais para Pessoas com Dispraxia, e na Educação Especial como um todo, sejam bem definidas por guidelines para orientação durante o desenvolvimento de aplicativos direcionados a estes sujeitos. Ainda há muito que ser determinado, testado e validado para que as interfaces gestuais sejam o mais amigáveis possíveis, assim como a captura dos movimentos e o reconhecimento de gestos com máxima significação.

Necessita-se, também, discutir o desenho da interface para este estilo de interação, promovendo estilos de aprendizagem individualizada ou colaborativa. Se há o propósito de igualdade de condições de fato para o aprendiz, cumprindo, assim, com uma premissa básica para caracterização das tecnologias assistivas.

Além disso, outros aspectos precisam ser investigados com maior profundidade pela comunidade de Informática na Educação, tais como a viabilidade financeira ao se desenvolverem aplicativos para tecnologias gestuais na EE, como também o nível de complexidade necessário para pessoas com Dispraxia utilizarem tais recursos e seus aspectos cognitivos mobilizados durante a alfabetização, aprendizagem, comunicação, intervenção ou terapia.

Trabalhos futuros seriam a disponibilização de aplicativos específicos para aprendizagem, comunicação, diversão e interação das pessoas com TDC nas várias tecnologias mencionadas, como ASUS Xtion Motion Sensor, Superfícies Interativas, Leap Motion, Kinect, MYO Armband, Nintendo Wii, PlayStation e Wisee, dentre outros.

\section{Agradecimentos}

Os autores agradecem à Universidade Federal do Piauí (UFPI) e ao Fundo Mackenzie de Pesquisa da Universidade Presbitariana Mackenzie (UPM).

\section{Referências}

Abbott, C. (2007). Defining assistive technologies-a discussion. Journal of Assistive Technologies, 1(1):6-9.

BDA (2012). Text to speech. The British Dyslexia Association. Disponível em: http://bdatech.org/whattechnology/text-to-speech/. Acessado em 18 de Maio de 2015.

Bohman, P. R. and Anderson, S. (2005). A conceptual framework for accessibility tools to benefit users with cognitive disabilities. In Proceedings of the 2005 International Cross-Disciplinary Workshop on Web Accessibility (W4A), pages 85-89. ACM.

BRASIL (1988). Constituição da República Federativa do Brasil: promulgada em 5 de outubro de 1988. Brasília-DF. Acessado em 11 de Maio de 2015. 
Cardoso, A. M. P., de Carvalho, R. C., da Silva, S. d. A. A., Pio, D. C., da Silveira, P. H. B. R., et al. (2014). Facil: Modelo para avaliação da literacia digital e informacional. Revista Brasileira de Informática na Educação, 22(03):46.

CAST (2012). About Universal Design for Learning. Disponível em: http://www.cast.org/udl/index.html. Acessado em 16 de Maio de 2015.

Chen, K.-W., Hsu, F.-C., Hsieh, Y.-Z., and Chou, C.-H. (2014). To design an interactive learning system for child by integrating blocks with kinect. In Global Engineering Education Conference (EDUCON), 2014 IEEE, pages 20-22. IEEE.

Clarke, M., Wright, J., Griffiths, T., and Price, K. (2011). A proposed framework for decision-making for assistive communication technology support: many perspectives, but one common goal. Journal of Assistive Technologies, 5(4):242-248.

Cohn, G., Morris, D., Patel, S. N., and Tan, D. S. (2011). Your noise is my command: sensing gestures using the body as an antenna. In Proceedings of the SIGCHI Conference on Human Factors in Computing Systems, pages 791-800. ACM.

CONVENÇÃO (2006). Convenção interamericana para a eliminação de todas as formas de discriminação contra as pessoas portadoras de deficiência. Acessado em 11 de Maio de 2015 .

Crawford, S. G., Wilson, B. N., and Dewey, D. (2001). Identifying developmental coordination disorder: consistency between tests. Physical \& occupational therapy in pediatrics, 20(2-3):29-50.

Davis, M., Dautenhahn, K., Powell, S., and Nehaniv, C. (2010). Guidelines for researchers and practitioners designing software and software trials for children with autism. Journal of Assistive Technologies, 4(1):38-48.

Erazo, O. and Pico, R. (2014). Interfaces de usuario basadas en gestos manuales sin contacto para la sala de clases: una revisión bibliográfica. Enfoque UTE, 5(4):pp-34.

Erazo, O., Pino, J. A., Pino, R., Asenjo, A., and Fernández, C. (2014). Magic mirror for neurorehabilitation of people with upper limb dysfunction using kinect. In System Sciences (HICSS), 47th Hawaii International Conference on, pages 2607-2615. IEEE.

Falcão, T. P. and Price, S. (2010). Informing design for tangible interaction: a case for children with learning difficulties. In Proceedings of the 9th International Conference on Interaction Design and Children, pages 190-193. ACM.

Feng, J., Lazar, J., Kumin, L., and Ozok, A. (2010). Computer usage by children with down syndrome: Challenges and future research. ACM Transactions on Accessible Computing (TACCESS), 2(3):13.

Foulder-Hughes, L. (2015). Free online bbc clips aid numeracy. Acessado em 17 de Maio de 2015.

Gil, A. C. (2002). Como elaborar projetos de pesquisa. São Paulo, 5:61.

Henderson, S. E., Sugden, D. A., Barnett, A. L., and Smits-Engelsman, C. (1992). Movement assessment battery for children. Psychological Corporation London.

IBGE, I. B. d. G. e. E. (2010). Censo demográfico 2010: características gerais da população, região e pessoas com deficiência. Acessado em 11 de Maio de 2015. 
Kleina, C. (2012). Tecnologia assistiva em educação especial e educação inclusiva. Curitiba: Intersaberes [livro eletrônico].

Kulessa, T. and Hoch, M. (1998). Efficient color segmentation under varying illumination conditions. IEEE Image and Multidimensional Digital Signal Processing Workshop.

McKnight, L. (2010). Designing for adhd in search of guidelines. In IDC 2010 Digital Technologies and Marginalized Youth Workshop.

McKnight, L. and Davies, C. (2012). Current perspectives on assistive learning technologies. The Kellogg College Centre for Research into Assistive Learning Technologies. Oxford University. December.

Mitra, S. and Acharya, T. (2007). Gesture recognition: A survey. Systems, Man, and Cybernetics, Part C: Applications and Reviews, IEEE Transactions on, 37(3):311-324.

Miyahara, M. and Möbs, I. (1995). Developmental dyspraxia and developmental coordination disorder. Neuropsychology Review, 5(4):245-268.

Nielsen, M., Störring, M., Moeslund, T. B., and Granum, E. (2004). A procedure for developing intuitive and ergonomic gesture interfaces for hci. In Gesture-Based Communication in Human-Computer Interaction, pages 409-420. Springer.

Norman, D. A. and Nielsen, J. (2010). Gestural interfaces: a step backward in usability. interactions, 17(5):46-49.

Olsen, J. Z., Fink, C., and Marxer, M. (2001). Handwriting without tears. Handwriting Without Tears Publisher.

Othman, M. F. and Keay-Bright, W. (2010). Using rotoscopy technique to assist the teaching of handwriting for children with dyspraxia. In Advances in Computer-Human Interactions, 2010. ACHI'10. Third International Conference on, pages 175-178. IEEE.

Othman, M. F. and Keay-Bright, W. (2011). Rotoscopy-handwriting prototype: Using computer animation technique to assist the teaching of handwriting for children with dyspraxia. In ITNG, pages 464-469.

Placitelli, A. P. and Gallo, L. (2012). Toward a framework for rapid prototyping of touchless user interfaces. In Complex, Intelligent and Software Intensive Systems (CISIS), 2012 Sixth International Conference on, pages 539-543. IEEE.

Portwood, M. (2013). Understanding developmental dyspraxia: A textbook for students and professionals. David Fulton Publishers.

Queck, F., Mysliwiec, T., and Zhao, M. (1995). Finger mouse: A freehand pointing interface. International Workshop on Automatic Face and Gesture Recognition, Zurich.

Salim, S. Y. (2014). Touchless vs direct-touch interface: Technical and applications. Interactive Multimedia Conference.

Shen, J., Luo, Y., Wang, X., Wu, Z., and Zhou, M. (2014). Gpu-based realtime hand gesture interaction and rendering for volume datasets using leap motion. International Conference on Cyberworlds, pages 85-92.

UNESCO, O. N. U. (2006). Convenção sobre os Direitos das Pessoas com Deficiência. Brasília-DF. Doc. A.61.611, Nova Iorque, 13 dez. Acessado em 12 de Maio de 2015. 